Applied Physiology, Nutrition, and Metabolism Physiologie appliquée, nutrition et métabolisme

\title{
Physiological and performance consequences of heavy thoracic load carriage in females
}

\begin{tabular}{|r|l|}
\hline Journal: & Applied Physiology, Nutrition, and Metabolism \\
\hline Manuscript ID & apnm-2016-0002.R1 \\
\hline Manuscript Type: & Article \\
\hline Complete List of Authors: & $\begin{array}{l}\text { Phillips, Devin; University of Alberta, Physical Education and Recreation } \\
\text { Stickland, Michael; University of Alberta } \\
\text { Petersen, Stewart; University of Alberta, }\end{array}$ \\
\hline Keyword: & $\begin{array}{l}\text { Heavy load carriage, Ventilation, Breathing pattern, Female, performance } \\
\text { < performance }\end{array}$ \\
\hline \multicolumn{2}{|c}{} \\
\hline \multicolumn{2}{|c}{} \\
\hline
\end{tabular}


Heavy load carriage in females.

\section{Physiological and performance consequences of heavy thoracic load carriage in females}

Devin B Phillips ${ }^{1}$

Michael K Stickland ${ }^{2,1}$

Stewart R Petersen ${ }^{1}$

${ }^{1}$ Faculty of Physical Education and Recreation, University of Alberta, Edmonton, AB, T6G $2 \mathrm{H} 9$

${ }^{2}$ Department of Medicine, University of Alberta, Edmonton, AB, T6G 2J3 and the G. F. MacDonald Centre for Lung Health, Covenant Health, Edmonton, AB, T5K 0L4.

Running head: Heavy load carriage in females

There are no conflicts of interest.

Correspondence

Stewart Petersen, $\mathrm{PhD}$

4-427 Van Vliet Complex

Faculty of Physical Education and Recreation

University of Alberta

Edmonton, AB,

T6G $2 \mathrm{H} 9$

Phone: (780) 4920996

Fax: (780) 4922364

Email: stewart.petersen@ualberta.ca 
Heavy load carriage in females.

\section{ABSTRACT}

The purposes of this experiment were to study physiological responses to graded exercise to exhaustion (Part I) and ventilatory responses during 45 minutes of exercise (Part II) with and without a $25 \mathrm{~kg}$ backpack. In Part I, on separate days, 24 females completed randomly-ordered modified Balke treadmill tests. Analysis revealed significant decreases in absolute $\dot{\mathrm{V}} \mathrm{O}_{2 \text { peak }}(3.5 \%)$, peak power output (20\%) and test duration (40\%) under load. There was a significant but modest negative relationship between body mass and the change in test duration between conditions $(r=-0.44)$. While physiological responses to peak exercise were similar, exercise performance was negatively impacted under load. On separate days in Part II, 14 females completed randomly-ordered, loaded and unloaded exercise challenges of submaximal treadmill walking at matched oxygen demands. Under load, breathing frequency, deadspace and minute ventilation were increased by $19.9,29.8$ and $11.6 \%(\mathrm{P}<0.05)$, respectively, while tidal volume and endinspiratory lung volume decreased by 13.6 and 6.0\% $(\mathrm{P}<0.05)$, respectively. After loaded exercise, maximal inspiratory pressure was decreased by $11.5 \% \quad(\mathrm{P}<0.05)$ with no changes in maximal expiratory pressure in either condition. Despite matched oxygen uptake between loaded and unloaded exercise challenges, perceived exertion and breathing discomfort were higher $(\mathrm{P}<0.05)$ under load. With heavy load carriage, the altered breathing pattern led to increased deadspace and minute ventilation, which likely contributed to higher perceptions of exercise stress and breathing discomfort. These results are similar to previous research in males and underscore the impact of heavy load carriage during exercise. 
Heavy load carriage in females.

Keywords: Heavy load carriage, Oxygen demand, Ventilation, Breathing pattern,

Female, Performance 
Heavy load carriage in females.

\section{Introduction}

Heavy load carriage is common and often essential to physically demanding occupations such as wildland firefighting, search and rescue and many military trades. In such occupations, arduous work is common and may require traversing rough terrain and steep inclines while carrying heavy loads in a time-sensitive matter. Fitted backpacks are often used to carry external loads of mission-essential equipment. Load mass is usually "absolute" rather than scaled according to body mass, stature or sex. While individual occupations may require unique mission-specific forms of load carriage, absolute loads of 25 - 30 kg are common (Knapik et al. 2004; Taylor et al. 2012; Epstein et al. 2013).

It has been well documented that heavy load carriage increases physiological strain and decreases exercise tolerance in young, healthy males (Louhevaara et al. 1995; Phillips et al. 2016b). Louhevaara et al. (1995) and Phillips et al. (2016b) investigated physiological responses and performance (test duration) during graded treadmill exercise in males with and without heavy load carriage. The load in the study by Louhevaara et al. was comprised of fire protective ensemble $(26 \mathrm{~kg})$ while in the study by Phillips et al., a weighted backpack $(25 \mathrm{~kg})$ was used. In both cases, peak physiological responses (e.g., oxygen uptake) were moderately reduced with much larger reductions demonstrated in exercise performance, indicated by test duration. Phillips et al. (2016b) reported that the difference in test duration (unloaded time minus loaded time) had a modest negative correlation with body mass. This observation suggests that heavier individuals may be more tolerant of work that involves carriage of heavy, absolute loads.

Thoracic load carriage with a backpack has been shown to alter pulmonary function and ventilatory mechanics during treadmill exercise at various durations and 
Heavy load carriage in females.

intensities (Muza et al. 1989; Dominelli et al. 2011; Faghy and Brown 2014; Phillips et al. 2016a). Collectively, these studies have reported that heavy load carriage altered breathing pattern and operating lung volume, and increased perceived exertion and breathing discomfort.

There is a relatively large body of work investigating physiological responses to load carriage however, almost exclusively in young males. For detailed reviews of sex differences and work performance readers are referred to Shephard and Bonneau (2003), Epstein et al. (2013) and Roberts et al. (2016). On average, females are smaller in mass, stature, lung volume and have lower functional capacity (Guennette et al. 2007; Epstein et al. 2013) and there are few reports of the effects of heavy load carriage in females. Notable exceptions are studies by Ruby et al. (2003) and Taylor et al. (2012). The former simulated an escape from a wildfire, with and without a $16 \mathrm{~kg}$ pack. While the transit time for both sexes was slower when carrying the pack, the effect was greater in females. Taylor et al. (2012) studied physiological responses and exercise tolerance during graded treadmill tests with and without fire protective ensemble $(\sim 20 \mathrm{~kg})$ in both male and female subjects. Physiological responses to peak exercise in the loaded condition were only slightly reduced for both sexes. However, exercise tolerance was greatly reduced for both sexes and more so for the female subjects.

The purposes of this study were twofold. The first purpose was to investigate the effects of heavy load carriage $(25 \mathrm{~kg}$ ) on physiological responses and performance (e.g., treadmill duration) during graded exercise in females. The second purpose was to study components of ventilation (e.g., breathing pattern, operating lung volume, calculated deadspace and alveolar ventilation) and perceptual responses during prolonged exercise 
Heavy load carriage in females.

with load carriage. Based on the results from our similar studies with males (Phillips et al. 2016 a and b), we hypothesized that heavy load carriage would substantially reduce graded treadmill exercise performance (power output and test duration) with only small reductions in peak ventilation and oxygen uptake. We also hypothesized that heavy load carriage would increase minute ventilation and perceived exertion during prolonged fixed-intensity submaximal exercise and the relative responses would be similar to our previous work in males.

\section{Methods}

Subjects

Twenty-four healthy, active females with no known history of asthma, smoking or cardiopulmonary disease provided informed consent to participate in the study, which had been previously approved by the appropriate research ethics board at the University of Alberta. Subjects were first screened for vigorous exercise with the Physical Activity Readiness Questionnaire (PAR-Q+) and secondly by a physician for safe ingestion of a temperature-transmitting capsule. The complex actions of hormonal fluctuations during the menstrual cycle are known to influence resting body temperature and ventilation (England and Farhi 1976; Hessemer and Bruck 1985). To minimize the effect of the hormones such as progesterone and estrogen on ventilatory responses to exercise, experimental trials were limited to the early follicular phase as reported by self-reported menstrual history (Lebrun et al. 1995; Guennette et al. 2007). The participants wore properly sized and fitted backpacks (Arc' Teryx Bora 75, North Vancouver BC) filled to a consistent weight $(25 \mathrm{~kg}$ ) and volume for all loaded trials throughout the experiment. 
Heavy load carriage in females.

The control condition involved unloaded exercise. In both conditions, participants were dressed in shorts, t-shirt and running shoes.

Design

The study was completed in two parts. Part I $(n=24)$ included two randomlyordered graded exercise tests to exhaustion in unloaded and loaded conditions. Part II $(n=14)$ included three distinct experimental steps in the following order: (1) randomlyordered pulmonary function tests in both unloaded and loaded conditions, (2) practice of the experimental protocols and (3) randomly-ordered prolonged exercise trials in unloaded and loaded conditions. All tests were carried out in an air-conditioned laboratory $\left(21-23^{\circ} \mathrm{C}\right)$ with low humidity $(1-10 \%)$.

\section{Sex comparison}

A subset of female subject data $(n=7)$ from this experiment was compared to a subset of 7 males from a previously published experiment (Phillips et al. 2016b). Male and female subjects from the two experiments were matched based on body mass and stature. Subsequent analysis compared differences in peak physiological responses and performance with and without a $25 \mathrm{~kg}$ pack between male and female data. It is important to note that data from both experiments were collected in the same laboratory following the same experimental protocols.

\section{Graded exercise tests}

On separate days subjects completed, in random order, a graded exercise test in both conditions to determine ventilatory threshold and measure $\mathrm{V}_{2 \text { peak }}$. The graded exercise test consisted of a constant speed $\left(1.5 \mathrm{~m} \cdot \mathrm{s}^{-1}\right)$ walking protocol on a motorized treadmill (Standard Industries, Fargo, ND). The test began at $0 \%$ grade and step increases 
Heavy load carriage in females.

of $2 \%$ grade were applied every 2 minutes until volitional exhaustion. Ventilatory threshold was identified by an increase in the ventilatory equivalent for oxygen $\left(\dot{\mathrm{V}}_{\mathrm{E}} / \dot{\mathrm{V}}_{2}\right)$ while the ventilatory equivalent for carbon dioxide $\left(\dot{\mathrm{V}}_{\mathrm{E}} / \dot{\mathrm{V}}_{\mathrm{CO}}\right)$ remained stable (Wasserman 1987).

A two-way breathing valve was used to collect expired gases (Hans Rudolph, Kansas City, MO, USA). Expired gases and ventilatory parameters were analyzed and calculated using a metabolic measurement system (TrueOne, ParvoMedics, Salt Lake City, UT, USA). The system was calibrated according to manufacturer's guidelines prior to each test. Calibration of the gas analyzers was verified immediately following each test. Heart rate was monitored continuously using telemetry and was recorded at the end of each minute (Polar Beat, Electro, Lachine, QC). After at least 24-hours of recovery, the subject completed the second graded exercise test in the alternate condition.

Power output during treadmill exercise was calculated using the equation: mass $\mathrm{x}$ speed $x$ grade. The mass used included the mass of the subjects and, in the loaded condition, also the mass of the pack.

Resting spirometry

Each subject completed randomly-ordered resting pulmonary function testing (TrueOne, ParvoMedics, Salt Lake City, UT, USA) in both unloaded and loaded conditions to determine forced vital capacity (FVC), forced expired volume in $1 \mathrm{~s}$ $\left(\mathrm{FEV}_{1}\right), \mathrm{FEV}_{1} / \mathrm{FVC}$ and peak expiratory flow rate (PEFR). Pulmonary function testing was performed according to the guidelines of the American Thoracic Society (Miller et al., 2005). In order to assess the effect of the pack mass on pulmonary function, the maneuvers were completed while standing upright with minimal forward lean. 
Heavy load carriage in females.

Spirometry was completed before and after the first graded exercise test to rule out exercise-induced bronchoconstriction (EIB) (Miller et al. 2005). No subjects were excluded from the experiment based on screening for EIB.

\section{Prolonged exercise practice session}

Prior to commencing the prolonged experimental trials, each subject completed a practice session, which accomplished several purposes. First, the exercise intensities (based on treadmill grade), determined from the results of the two graded exercise tests were evaluated to ensure that the oxygen demand of the loaded and unloaded conditions was satisfactorily matched $\left( \pm 0.1 \mathrm{~L} \cdot \mathrm{min}^{-1}\right)$. Second, the subject exercised for 20 minutes in each condition to evaluate tolerance for the planned experimental exercise challenges. Third, the subject was fully oriented to all experimental procedures (e.g., measurement of inspiratory capacity during exercise, measurement of maximal voluntary inspiratory and expiratory pressures before and after exercise).

The specific exercise intensities for the prolonged trials were determined using linear regression; following previously reported methods (Phillips et al. 2016a). For the loaded condition, the treadmill grade was set at $2 \%$ below the grade that elicited ventilatory threshold during the loaded graded exercise test. This $\dot{\mathrm{V}} \mathrm{O}_{2}$ value $\left(\mathrm{Lmin}^{-1}\right)$ was considered the "target $\mathrm{V}_{2}$ " for the prolonged exercise trials. Linear regression established the relationship between absolute oxygen uptake and treadmill grade from the unloaded test, and subsequently, the regression equation was used to solve for unloaded grade that corresponded to the target $\dot{\mathrm{V}} \mathrm{O}_{2}$. Treadmill speed was the same during the graded exercise test and remained constant $\left(1.5 \mathrm{~m} \cdot \mathrm{s}^{-1}\right)$ throughout the prolonged exercise bouts for both conditions. 
Heavy load carriage in females.

\section{Prolonged exercise trial}

Subjects were randomly assigned to either the loaded or unloaded condition for the first experimental prolonged trial. During the protocol, subjects completed 45 minutes of exercise at a constant speed $\left(1.5 \mathrm{~m} \cdot \mathrm{s}^{-1}\right)$ and grade. As described above, the treadmill grade for each condition was pre-determined and verified during the practice to ensure that $\dot{\mathrm{V}} \mathrm{O}_{2}$ was matched between conditions. Subjects completed a brief warm-up and cooldown before and after exercise. Open-circuit spirometry provided continuous measurement of respiratory gas exchange during the protocol. Inspiratory capacity maneuvers were completed at baseline and at 5-minute intervals during exercise. Perceived exercise stress, breathing discomfort and leg fatigue was recorded at the conclusion of the first 5-minute measurement cycle and was repeated every 10 minutes throughout the experimental trial. Heart rate was recorded every minute and blood pressure was taken by auscultation at rest and at 5, 25 and 45 minutes of exercise.

\section{Deadspace ventilation}

Deadspace ventilation $\left(\dot{\mathrm{V}}_{\mathrm{D}}\right)$ was determined from the difference between minute ventilation and alveolar ventilation $\left(\dot{\mathrm{V}}_{\mathrm{A}}\right)$ as described by West (2000). Alveolar ventilation was estimated using:

$$
\dot{\mathrm{V}}_{\mathrm{A}}=\left(\dot{\mathrm{VCO}}_{2} / \mathrm{P}_{\mathrm{a}} \mathrm{CO}_{2}\right) \cdot \mathrm{K}
$$

In this equation, $\mathrm{K}$ is a conversion factor used to adjust $\mathrm{VCO}_{2}$ to body temperature and pressure. Due to the difficulty of determining arterial carbon dioxide partial pressure $\left(\mathrm{P}_{\mathrm{a}} \mathrm{CO}_{2}\right)$, pressure of end-tidal carbon dioxide $\left(\mathrm{P}_{\mathrm{ET}} \mathrm{CO}_{2}\right)$ was assumed to be equal to $\mathrm{P}_{\mathrm{a}} \mathrm{CO}_{2}$ (Stickland et al. 2013). 
Heavy load carriage in females.

End-tidal $\mathrm{CO}_{2}$ was measured (R-1 pump, P-61B sensor and CD-3A CO 2 analyzer, AEI technologies Naperville, IL, USA) from a small port off of the mouthpiece collected through a drying line. End-tidal $\mathrm{CO}_{2}$ data were recorded with a data acquisition system (Powerlab 8/35, AD Instruments, New South Wales, Australia) and stored for subsequent analysis.

\section{Lung Volume}

Changes in operating lung volume were estimated from measurements of inspiratory capacity (IC) taken at rest and at 5-minute intervals during exercise. An inspiratory pneumotach (ParvoMedics, Sandy, UT, USA) attached to the two-way breathing valve was used to measure inspired volume. End-expiratory lung volume was calculated by subtracting IC from FVC obtained at rest and it was assumed that total lung capacity did not change throughout exercise (Johnson et al. 1999). End-inspiratory lung volume was then estimated by adding $\mathrm{V}_{\mathrm{T}}$ and EELV. Tidal volume was recorded in the minute leading up to the IC maneuver and then averaged. Changes in EELV and EILV were expressed as a percentage of FVC. The determination of operating lung volume is dependent on the correct performance and analysis of the IC maneuver. Subjects were given a verbal prompt to complete the IC maneuver up to total lung capacity. To improve the likelihood of valid and reliable results, each subject had extensive practice of the IC maneuver during the practice session prior to the experimental protocols. During analysis, volume was corrected for any pneumotachometer drift that may have occurred by selecting six breaths prior to the maneuver and zeroing expiratory volume (Johnson et al. 1999; Dominelli et al. 2011). 
Heavy load carriage in females.

\section{Maximal voluntary respiratory muscle pressures}

Maximal voluntary inspiratory pressure (MIP) and maximal voluntary expiratory pressure (MEP) were measured as indices of respiratory muscle strength before prolonged exercise and within 4 minutes of exercise completion. Maximal inspiratory and expiratory pressures were measured by having the subject produce a maximal inspiration or expiration through a mouthpiece into an occluded rigid tube (Phillips et al. 2016a). A small leak (1 mm diameter) was used to prevent glottis closure. The tube was attached to a positive or negative pressure gauge (Cole Parmer Co, Stratford, USA). Measurement of MIP was initiated at residual volume (RV) and MEP at total lung capacity. Measurements were repeated until there were two maximal values within $5 \mathrm{~cm} \mathrm{H}_{2} \mathrm{O}$. It is well known that maximal volitional pressures are highly dependent on subject motivation and effort. However, each subject had a comprehensive familiarization and numerous opportunities to practice the technique prior to the experimental trials.

\section{Perceptual responses}

Perceived exercise stress, breathing discomfort and leg fatigue were measured using three separate 11-point systems during the prolonged trial. The scales were anchored such that 0 represented "No exercise stress, breathing discomfort or leg fatigue" and 10 represented "Maximal exercise stress, breathing discomfort or leg fatigue." (Borg 1982; O’Donnell et al. 2000; Phillips et al. 2016a).

\section{Core temperature}

Core temperature was monitored continuously by telemetry and recorded at 5minute intervals during the prolonged experimental trials (VitalSense ${ }^{\mathrm{TM}}$, Mini Mitter, 
Heavy load carriage in females.

Bend, OR). The temperature capsule was ingested 5 hours prior to the start of the experimental trial.

Data analysis

Data are presented as mean \pm standard error (SE) unless otherwise indicated. For part I, student's t-test was used to detect any differences in ventilatory threshold and peak exercise data between conditions. Unpaired t-test analysis was used to compare variables of interest between female and male groups. Pearson Product-Moment correlation was used to examine relationships between variables of interest. For Part II, a two-way, repeated-measures analysis of variance was used to compare loaded and unloaded conditions on key dependent variables at rest and 5-minute intervals during the prolonged exercise trials. If a significant change or interaction effect was found, Tukey's post hoc was used to locate each difference. Student's t-test was used to detect any differences in pulmonary function, MIP and MEP data between conditions. All statistical analyses were performed using Sigma Plot Software version 12.0 (Systat Software Inc., Chicago, USA). Significance was set a priori at $\mathrm{P}<0.05$.

\section{Results}

\section{Subject characteristics}

Table 1 shows subject characteristics for Parts I $(n=24)$ and II $(n=14)$. There were no differences in mass, stature, body mass index or body surface area. The backpack represented approximately $32-48 \%$ of the load carried relative to body mass across the group. 
Heavy load carriage in females.

\section{Resting pulmonary function}

All subjects had normal lung function $\left(\mathrm{FEV}_{1}>80 \%\right.$ predicted) in the unloaded condition with no evidence of obstructive or restrictive patterns (Table 2). In the loaded condition, FVC and $\mathrm{FEV}_{1}$ were significantly reduced when compared to the unloaded condition. Peak expiratory flow and the $\mathrm{FEV}_{1} / \mathrm{FVC}$ ratio were not different between conditions.

\section{Graded exercise performance and physiological responses}

The physiological responses to graded exercise are shown in Table 2. In the loaded condition, both the absolute $\dot{\mathrm{VO}}_{2}$ and the $\dot{\mathrm{V}} \mathrm{O}_{2}$ relative to total mass were significantly reduced at ventilatory threshold and peak exercise when compared to the unloaded condition. Minute ventilation was not different between conditions at ventilatory threshold and peak exercise although breathing pattern was affected at both physiological milestones (Table 2). Power output in the loaded condition was significantly reduced by $41 \%$ at ventilatory threshold and by $20 \%$ at peak exercise compared to the unloaded condition. Test duration was decreased by approximately $39 \%$ in the loaded condition (Table 2). There were no significant correlations between selected indices of size, aerobic fitness or pulmonary function and the changes in $\dot{\mathrm{V}} \mathrm{O}_{2}$, minute ventilation and power output at peak exercise between loaded and unloaded conditions. There was however, a significant moderate negative correlation between the change in test duration between conditions, and body mass $(r=-0.44)$.

\section{Sex comparison}

Table 3 shows subject characteristics and selected metabolic and performance variables from the graded exercise tests in the subsets of females $(n=7)$ and males $(n=7)$. 
Heavy load carriage in females.

Body mass and stature were identical between the groups, however, the males had higher peak $\dot{\mathrm{VO}_{2}}$, minute ventilation and test duration with and without load carriage. The relative reductions in peak physiological responses and treadmill test duration under load were similar between the groups.

\section{Prolonged exercise trial}

Table 4 shows selected physiological responses during the prolonged exercise trials. Oxygen demand was the same between conditions (approximately $67 \% \dot{\mathrm{VO}}_{2 \text { peak }}$ ) throughout the exercise trials. Carbon dioxide production, core temperature and blood pressure were the same between conditions at every time point. Minute ventilation was significantly higher in the loaded condition throughout the experimental trial despite maintenance of alveolar ventilation (Fig 1). The difference in minute ventilation between conditions became greater over time and was likely secondary to a progressive increase in deadspace ventilation in the loaded condition (Fig 1). The increase in deadspace ventilation under load was the result of altered breathing pattern (decreased $V_{T}$ and increased in $f_{B}$, both $\left.p<0.05\right)$ compared to the unloaded condition. The change in deadspace ventilation in the loaded trial varied widely among subjects from $6-70 \%(0.5$

- 13.0 $\left.\mathrm{Lmin}^{-1}\right)$ but there were no associations between the change in $\left(\dot{\mathrm{V}}_{\mathrm{D}}\right)$ and either body mass, height, $\mathrm{FEV}_{1}$ or $\dot{\mathrm{VO}}_{2 \text { peak }}$.

Figure 2 shows operating lung volumes in both conditions throughout the prolonged exercise trial. At rest, loaded EELV was slightly lower $(\mathrm{P}<0.05)$ then unloaded. Compared to resting values, EILV increased and EELV decreased during exercise in both conditions. However, EILV in the loaded condition was significantly lower than unloaded with no changes in EELV, between conditions, at all time points 
Heavy load carriage in females.

during the prolonged exercise trial. These data suggest that the decrease in $\mathrm{V}_{\mathrm{T}}$ during steady-state exercise under load was the result of a lower EILV.

Perceived exercise stress was greater $(\mathrm{P}<0.05)$ in the loaded condition at every time point despite the matched $\mathrm{V}_{2}$. Perceived breathing discomfort and leg fatigue became significantly greater in the loaded condition during the final 20 minutes of exercise (Table 4).

Before prolonged exercise, there were no differences between conditions in MIP and MEP values (Table 5). After loaded exercise, MIP was decreased $(\mathrm{p}<0.05)$ when compared to the pre-exercise value. There was no change in MEP values in either condition after prolonged exercise.

\section{Discussion}

\section{Major findings}

The principal findings of this study are threefold. First, in the loaded condition, peak absolute $\dot{\mathrm{VO}}_{2}$ and minute ventilation were only moderately reduced while much larger decreases in variables indicative of exercise performance (power output and test duration) were observed during the graded exercise tests. The reduction in loaded test duration, compared to unloaded, during graded exercise was moderately associated with body mass. Second, during 45 minutes of strenuous exercise with heavy thoracic load carriage, minute ventilation, perceived exercise stress and breathing discomfort were increased when compared to unloaded exercise at matched oxygen demand. Third, it appears the relative changes in physiological responses between thoracic loading and unloaded control conditions, from both graded and prolonged fixed-intensity exercise 
Heavy load carriage in females.

tests in females are similar to previous work with males (Louhevaara et al. 1995; Taylor et al. 2012; Phillips et al. 2016a; Phillips et al. 2016b). Present results suggest that with heavy load carriage, reductions in physiological responses at peak exercise were similar, regardless of sex. The reductions in graded exercise performance (e.g., power output and test duration) under load were also similar. The similar, modest negative relationships between the difference in test duration and body mass for both males (Phillips et al. 2016b) and females (present data) suggest that work tolerance under load is moderately influenced by size for both sexes. Of note, between-sex comparisons are made cautiously as the male and female data came from separate experiments and the female sample size was rather small.

Part I

Maximal exercise tests

Reductions in exercise performance and physiological maxima have been previously demonstrated in both males and females during graded treadmill exercise with load carriage consisting of fire protective ensemble weighing approximately $20 \mathrm{~kg}$ (Taylor et al. 2012). However, our findings are the first to demonstrate these responses in females with heavy thoracic load carriage using a fitted backpack. Other researchers have suggested that the pack straps might contribute to restriction of chest wall expansion (Louhevaara et al. 1995; Eves et al. 2005), however, with a correctly sized and properly fitted modern backpack, the load is located on the posterior aspect of the thorax, and much of the weight is borne by the hip belt. The resulting respiratory mechanics are likely quite different, and thus while posterior thoracic loading and chest wall strapping might have similar outcomes (e.g., attenuated ventilation) the underlying reasons may be 
Heavy load carriage in females.

quite different. Although $\dot{\mathrm{V}}_{2 \text { peak }}$ was slightly reduced with load in the current study, it is difficult to conclude that the reduction in peak oxygen uptake was secondary to attenuated exercise ventilation with thoracic loading. Breathing pattern at peak exercise was more rapid and shallow with the pack compared to the unloaded condition, which is suggestive of increased wasted ventilation. Additional research will be required to identify the mechanism(s) leading to decreased $\dot{\mathrm{V}}_{2 \text { peak }}$ with heavy thoracic load carriage.

Logically, $\dot{\mathrm{V}} \mathrm{O}_{2}$ should be slightly elevated while standing at rest under load, but the small change in $\dot{\mathrm{V}} \mathrm{O}_{2 \text { peak }}$ suggests that $25 \mathrm{~kg}$ thoracic loading does not severely impair the physiological reserve (peak - baseline). During load carriage, there is an expected increase in metabolic rate in order to support the additional weight and lift it against gravity. The increased metabolic rate may be predicted $\left(17 \mathrm{ml} \mathrm{O}_{2} \mathrm{x}\right.$ load mass: see Taylor et al. 2012) however the effect is not constant and has been shown to increase with treadmill grade (Phillips et al. 2016b; Taylor et al. 2016). The increased oxygen demand of load carriage will effectively lower the energy available for locomotion (Wang et al. 2004; Taylor et al. 2012). Our results suggest that the decrease in treadmill performance under load is partially explained by how total oxygen consumption is partitioned into maintenance of internal function, locomotion and load carriage (Taylor et al. 2012). Although maximal exercise is not a common task in occupations such as infantry and firefighting, it is important to understand the potential performance limitations when carrying heavy loads during critical tasks such as search and rescue or emergency escape situations (Ruby et al. 2003). For many emergency response occupations, prolonged work at or near the ventilatory threshold is particularly relevant for time-sensitive operational effectiveness. The large decrease in power output at ventilatory threshold 
Heavy load carriage in females.

under load underscores the impact of load carriage on work performance at this physiological milestone. The decrease in ambulatory reserve under load could have serious implications for occupational safety and effectiveness.

\section{Sex comparison}

Results of the comparison of graded exercise test results in a subset of female subjects with a size-matched group of males (Phillips et al. 2016b) revealed that, although males had higher $\dot{\mathrm{V}} \mathrm{O}_{2 \text { peak }}$ and greater test duration, the relative reductions in test time and $\dot{\mathrm{V}} \mathrm{O}_{2 \text { peak }}$ with load carriage were similar between groups (Table 3 ). The large reduction in treadmill test duration under load appears to be at least partially explained by body mass in young females and is similar to previously reported associations between performance and body mass in males (Bilzon et al. 2001; Phillips et al. 2016b). Present results are in agreement with previous work reporting lower aerobic power and work capacity during unloaded exercise in females, compared to males (McClaran et al. 1998; Guenette et al. 2007), however, we are the first to report the changes in performance with and without $25 \mathrm{~kg}$ thoracic load carriage. Our results suggest that body mass may be a stronger predictor of load carriage performance than sex because the change in test duration was the same for size-matched males and females. We recognize the limitation of such analysis and recognize that planned comparisons with greater statistical power are required to properly address these questions. Future research where large heterogeneous samples of males and females are systematically matched on variables such as mass, stature, total lung capacity or aerobic fitness would be helpful to better understand the implications of heavy load carriage on between-sex differences for male and female workers. 
Heavy load carriage in females.

\section{Part II}

\section{Prolonged load carriage at matched oxygen demand}

The findings in this investigation are important for understanding the ventilatory response to thoracic load carriage in females. There is previous work showing differences between females and males during unloaded exercise, however, adding thoracic load carriage can impose a new set of respiratory constraints (Muza et al. 1989; Guenette et al. 2007; Dominelli et al. 2011; Phillips et al. 2016a). Although females have smaller lungs and breathe at lower absolute ventilation rates compared to males, females may require a larger relative recruitment of respiratory muscles to support exercise with thoracic load carriage and maintain alveolar ventilation. Guenette et al (2007) reported dramatically increased work of breathing in young trained females above ventilation rates of 60 $\mathrm{L} \cdot \min ^{1}$, compared to males, during incremental unloaded cycle ergometer exercise. The authors suggested that the smaller airways of females may result in more turbulent flow and hence resistive work of breathing would be increased, at lower ventilation rates, to overcome this anatomical difference. In the current study, ventilation rates exceeded 60 $\mathrm{L} \cdot \mathrm{min}^{-1}$ under load at approximately 15 minutes of sustained treadmill exercise. Minute ventilation under load was always significantly higher than during the unloaded control and the difference between conditions increased over time throughout the exercise trial. The increased minute ventilation was the result of an alteration in breathing pattern (more rapid and shallow) and was likely an adjustment to minimize the elastic work of breathing during sustained load carriage at a fixed treadmill speed and grade (45 min). However, the progressive increase in deadspace ventilation, over time, furthered the increase in minute ventilation and also breathing discomfort. Previous research has 
Heavy load carriage in females.

reported alterations in ventilatory responses during exercise in young females (McClaran et al. 1998; Guenette et al. 2007); however, we are the first to compare unloaded and loaded ventilatory responses during prolonged fixed intensity exercise. During the prolonged exercise trial $\dot{\mathrm{VO}}_{2}$, core temperature and blood pressure were the same between conditions at every time point. This was an important methodological step in our experiment to ensure any observed alterations in ventilation under load were not the result of altered metabolic, thermal or hemodynamic state.

Although we did not measure intra-thoracic pressure, we suggest that work of breathing would logically have been elevated in the loaded condition. The $12 \%$ reduction in post-exercise MIP is suggestive of inspiratory muscle fatigue and is larger than our previous findings (7\% reduction) in males using the same experimental protocol (Phillips et al. 2016b). On average, the females in this study were smaller in size (mass, stature and lung volume) and it is possible the increased relative load, from the pack, contributed to the reduction in inspiratory muscle strength.

Breathing discomfort was increased in the loaded condition and is likely secondary to the increased minute ventilation during exercise. These findings have serious implications for emergency response and military occupations. If the work is time sensitive and prolonged exercise is required, the increased perceived exercise and breathing discomfort, secondary to increased ventilation could require adjustments in external work (speed and/or grade) and decreased occupational effectiveness.

\section{Future research}

The results from this study indicate that, although treadmill performance is greatly decreased, physiological maxima and the physiological reserve during graded exercise to 
Heavy load carriage in females.

exhaustion are relatively well preserved during exercise with heavy thoracic load carriage in young females. Our previous study with male subjects revealed similar results. The modest influence of body size on exercise tolerance with heavy loads appears to be consistent for both sexes. However, these observations are made with caution because the subjects in both experiments were convenience samples. In order to fully explore this question, further research where males and females are matched on specific characteristic (e.g., mass, height, lung function, aerobic fitness) with larger sample sizes is required.

The altered ventilatory responses under load during prolonged exercise (Part II) suggest increased total work of breathing. These data are similar to previous load carriage studies with males (Phillips et al. 2016a). However, this remains speculative until confirmed by measurements of work of breathing during prolonged exercise with heavy thoracic load carriage. Further research is required to understand how ventilatory mechanics and lung compliance are altered under load.

\section{Conclusion}

This study reported the physiological and performance consequences of heavy load carriage in females during both graded exercise to exhaustion and prolonged submaximal exercise. Our findings suggest that the physiological response to graded exercise under load is similar in males and females. Although load carriage performance, as indicated by treadmill test duration, is moderately associated with body size, other factors must also contribute to work capacity under load. Understanding how load carriage affects physiological responses and work performance in both males and females can improve the validity of physiological testing for occupations that require heavy load carriage. 
Heavy load carriage in females.

\section{Conflict}

The authors declare that there are no conflicts of interest.

\section{Acknowledgments}

The technical assistance from Eric Bell, Cameron Ehnes, Lauren Lee and Bradley Welch is appreciated. 
Heavy load carriage in females.

\section{References}

Bilzon, J.L., Allsopp, A.J., and Tipton, M.J. 2001. Assessment of physical fitness for occupations encompassing load-carriage tasks. Occup. Med. (Lond). 51(5): 357-361.

Borg, G.A. 1982. Psychophysical bases of perceived exertion. Med. Sci. Sports Exercise. 14(5): 377-381.

Dominelli, P.B., Sheel, A.W., and Foster, G.E. 2011. Effect of carrying a weighted backpack on lung mechanics during treadmill walking in healthy men. Eur. J. Appl. Physiol. doi: 10.1007/s00421-011-2177-8.

England, S.J., and Farhi, L.E. 1976. Fluctuations in alveolar $\mathrm{CO}_{2}$ and in base excess during the menstrual cycle. Respir. Physiol. 26(2): 157-161.

Epstein, Y., Yanovich, R., Moran, D.S., and Heled, Y. 2013. Physiological employment standards IV: integration of women in combat units physiological and medical considerations. Eur. J. Appl. Physiol. 113(11): 2673-2690. doi: 10.1007/s00421-0122558-7.

Eves, N.D., Jones, R.L., and Petersen, S.R. 2005. The influence of the self-contained breathing apparatus (SCBA) on ventilatory function and maximal exercise. Can. J. Appl. Physiol. 30(5): 507-519.

Faghy, M.A., and Brown, P.I. 2014. Thoracic load carriage-induced respiratory muscle fatigue. Eur. J. Appl. Physiol. 114(5): 1085-1093. doi: 10.1007/s00421-014-2839-4.

Guenette, J.A., Witt, J.D., McKenzie, D.C., Road, J.D., and Sheel, A.W. 2007.

Respiratory mechanics during exercise in endurance-trained men and women. J. Physiol. 581(3): 1309-1322. doi: 10.1113/jphysiol.2006.126466.

Hessemer, V., and Brück, K. 1985. Influence of menstrual cycle on thermoregulatory, metabolic, and heart rate responses to exercise at night. J. Appl. Physiol. 59(6): 1911-1917.

Johnson, B.D., Weisman, I.M., Zeballos, R.J., and Beck, K.C. 1999. Emerging concepts in the evaluation of ventilatory limitation during exercise: the exercise tidal flowvolume loop. Chest 116(2): 488-503.

Knapik, J.J., Reynolds, K.L., and Harman, E. 2004. Soldier load carriage: historical, physiological, biomechanical, and medical aspects. Mil. Med. 169(1): 45-56.

Lebrun, C., McKenzie, D., and Prior, J. 1995. Effects of menstrual cycle phase on athletic performance. Med. Sci. Sports Exercise. 27: 437-444. 
Heavy load carriage in females.

Louhevaara, V., Ilmarinen, R., Griefahn, B., Künemund, C., and Mäkinen, H. 1995. Maximal physical work performance with European standard based fire-protective clothing system and equipment in relation to individual characteristics. Eur. J. Appl. Physiol. 71(2-3): 223-229.

McClaran, S.R., Harms, C.A., Pegelow, D.F., and Dempsey, J.A. 1998. Smaller lungs in women affect exercise hyperpnea. J. Appl. Physiol. 84(6): 1872-1881.

Miller, M.R., Hankinson, J., Brusasco, V., Burgos, F., Casaburi, R., Coates, A., Crapo, R., Enright, P., van der Grinten, C.P.M., Gustafsson, P., Jensen, R., Johnson, D.C., Macintyre, N., McKay, R., Navajas, D., Pedersen, O.F., Pellegrino, R., Viegi, G., Wanger, J., ATS/ERS Task Force. 2005, August. Standardisation of spirometry. European Respiratory Society. doi: 10.1183/09031936.05.00034805.

Muza, S.R., Latzka, W.A., Epstein, Y., and Pandolf, K.B. 1989. Load carriage induced alterations of pulmonary function. Int. J. Indust. Ergonomics 3(3): 221-227. doi: 10.1016/0169-8141(89)90021-8.

O'Donnell, D.E., Hong, H.H., and Webb, K.A. 2000. Respiratory sensation during chest wall restriction and dead space loading in exercising men. J. Appl. Physiol. 88(5): 1859-1869.

Phillips, D.B., Stickland, M.K., and Petersen, S.R. 2016a. Ventilatory responses to prolonged exercise with heavy load carriage. Eur. J. Appl. Physiol. 116(1): 19-27. doi: 10.1007/s00421-015-3240-7.

Phillips D.B., Stickland, M.K., Lesser, I.A., and Petersen, S.R. 2016b. The effects of heavy load carriage on physiological responses to graded exercise. Eur. J. Appl. Physiol. 116(2): 275-280. doi: 10.1007/s00421-015-3280-z.

Roberts, D., Gebhardt, D.L., Gaskill, S., Borges, A.S., Roy, T.C., and Sharp, M.A. 2016. Sex, human performance and physical employment standards. Appl. Physiol. Nutr. Metab. (In press)

Ruby B., Leadbetter G., Armstrong D., Gaskill S. 2003. Wildland firefighter load carriage: effects on transit time and physiological responses during simulated escape to safety zone. Int. J. Wildland Fire. 12:111-116.

Sheel, A.W., and Romer, L.M. 2012. Ventilation and respiratory mechanics. Compr. Physiol. 2(2): 1093-1142. doi: 10.1002/cphy.c100046.

Shephard, R.J., and Bonneau, J. 2002 Assuring gender equity in recruitment standards for police officers, Can. J. Appl. Physiol. 27(3): 263-295 
Heavy load carriage in females.

Stickland, M.K., Lindinger, M.I., Olfert, I.M., Heigenhauser, G.J.F., and Hopkins, S.R. 2013. Pulmonary gas exchange and acid-base balance during exercise. Compr.

Physiol. 3(2): 693-739. doi: 10.1002/cphy.c110048.

Taylor, N.A.S., Lewis, M.C., Notley, S.R., and Peoples, G.E. 2012. A fractionation of the physiological burden of the personal protective equipment worn by firefighters. Eur. J. Appl. Physiol. 112(8): 2913-2921. doi: 10.1007/s00421-011-2267-7.

Taylor, N.A.S., Peoples, G.E., and Petersen, S.R. 2016. Load carriage, human performance and physiological employment standards. Appl. Physiol. Nutr. Metab. In press.

Wang, L.-Y., and Cerny, F.J. 2004. Ventilatory response to exercise in simulated obesity by chest loading. Med. Sci. Sports Exercise. 36(5): 780-786.

Wasserman, K. 1987. Determinants and detection of anaerobic threshold and consequences of exercise above it. Circulation. 76(6 Pt 2): VI29-39.

West JB (ed). 2008. Respiratory physiology: the essentials, 8th ed. Baltimore, MD. 
Heavy load carriage in females.

Table 1. Mean $( \pm \mathrm{SD})$ subject characteristics

\begin{tabular}{cccc}
\hline Characteristic & Mean & SD & Range \\
\hline $\begin{array}{c}\text { Part I (n=24) } \\
\text { Age }(\mathrm{y})\end{array}$ & 24 & 4 & $18-33$ \\
Height $(\mathrm{cm})$ & 169 & 7 & $158-184$ \\
Mass $(\mathrm{kg})$ & 65.5 & 6.1 & $51.6-78.3$ \\
BMI $\left(\mathrm{m} \cdot \mathrm{kg}^{-2}\right)$ & 22.9 & 2.4 & $19.1-30.8$ \\
BSA $\left(\mathrm{m}^{2}\right)$ & 1.76 & 0.08 & $1.50-1.95$ \\
Part II $(\mathbf{n}=\mathbf{1 4})$ & & & $18-33$ \\
Age $(\mathrm{y})$ & 24 & 4 & $162-178$ \\
Height $\left(\mathrm{cm}^{2}\right)$ & 170 & 5 & $58.7-78.0$ \\
Mass $\left(\mathrm{kg}^{2}\right)$ & 65.1 & 6.5 & $20.0-26.5$ \\
BMI $\left(\mathrm{m} \cdot \mathrm{kg}^{-2}\right)$ & 22.5 & 1.8 & $1.65-1.93$ \\
BSA $\left(\mathrm{m}^{2}\right)$ & 1.75 & 0.09 & \\
\hline
\end{tabular}

BMI, body mass index; BSA, body surface area. 
Heavy load carriage in females.

Table 2. Mean $( \pm \mathrm{SE})$ graded exercise responses (Part I) and resting pulmonary function (Part II)

\begin{tabular}{|c|c|c|}
\hline & Unloaded & Loaded \\
\hline \multicolumn{3}{|c|}{ Ventilatory Threshold $(n=24)$} \\
\hline$\dot{\mathrm{V}} \mathrm{O}_{2}\left(\mathrm{~L} \cdot \mathrm{min}^{-1}\right)$ & $2.00(0.08)$ & $1.93(0.07)^{a}$ \\
\hline$\dot{\mathrm{V}} \mathrm{O}_{2}\left(\mathrm{ml} \cdot \mathrm{kg}_{\text {total mass }}{ }^{-1} \cdot \mathrm{min}^{-1}\right)$ & $30.5(0.9)$ & $21.2(0.6)^{a}$ \\
\hline$\dot{\mathrm{V}}_{\mathrm{E}}\left(\mathrm{L} \cdot \min ^{-1}\right)$ & $57.2(2.7)$ & $56.4(2.4)$ \\
\hline$f_{B}\left(\right.$ breaths $\left.\cdot \min ^{-1}\right)$ & $29(1)$ & $33(1)^{a}$ \\
\hline $\mathrm{V}_{\mathrm{T}}(\mathrm{L})$ & $1.88(0.08)$ & $1.73(0.09)^{a}$ \\
\hline Heart Rate (bpm) & $162(3)$ & $160(2)$ \\
\hline Power output (W) & $108(6)$ & $64(7)^{a}$ \\
\hline \multicolumn{3}{|l|}{ Peak Exercise $(n=24)$} \\
\hline$\dot{\mathrm{V}} \mathrm{O}_{2}\left(\mathrm{~L} \cdot \mathrm{min}^{-1}\right)$ & $2.83(0.12)$ & $2.73(0.11)^{a}$ \\
\hline$\dot{\mathrm{V}} \mathrm{O}_{2}\left(\mathrm{ml} \cdot \mathrm{kg}_{\text {total mass }}{ }^{-1} \cdot \mathrm{min}^{-1}\right)$ & $42.8(1.6)$ & $30.1(1.0)^{a}$ \\
\hline$\dot{\mathrm{V}}_{\mathrm{E}}\left(\mathrm{L} \cdot \min ^{-1}\right)$ & $112.4(4.1)$ & $109.3(4.2)$ \\
\hline $\mathrm{f}_{\mathrm{B}}\left(\right.$ breaths $\left.\cdot \min ^{-1}\right)$ & $50(2)$ & $55(2)^{a}$ \\
\hline $\mathrm{V}_{\mathrm{T}}(\mathrm{L})$ & $2.26(0.08)$ & $2.02(0.09)^{a}$ \\
\hline RER & $1.15(0.01)$ & $1.16(0.01)$ \\
\hline Heart Rate (bpm) & $190(3)$ & $189(2)$ \\
\hline Power output (W) & $183(8)$ & $146(8)^{a}$ \\
\hline Time (s) & $1197(40)$ & $726(32)^{a}$ \\
\hline \multicolumn{3}{|l|}{ Pulmonary Function $(n=14)$} \\
\hline $\mathrm{FVC}(\mathrm{L})$ & $4.47(0.16)$ & $4.20(0.17)^{a}$ \\
\hline $\mathrm{FEV}_{1}(\mathrm{~L})$ & $3.60(0.09)$ & $3.44(0.10)^{a}$ \\
\hline $\mathrm{FEV}_{1} / \mathrm{FVC}$ & $0.81(0.02)$ & $0.82(0.02)$ \\
\hline $\operatorname{PEFR}\left(\mathrm{L} \cdot \mathrm{s}^{-1}\right)$ & $8.07(0.29)$ & $7.82(0.31)$ \\
\hline \multicolumn{3}{|c|}{$\begin{array}{l}\dot{\mathrm{V}} \mathrm{O}_{2} \text {, oxygen consumption; } \dot{\mathrm{V}}_{\mathrm{E}} \text {, minute ventilation; } \mathrm{f}_{\mathrm{B}} \text {, breathing frequency; } \mathrm{V}_{\mathrm{T}} \text {, tidal } \\
\text { volume; RER, respiratory exchange ratio; } \mathrm{FVC} \text {, forced vital capacity; } \mathrm{FEV}_{1} \text {, forced } \\
\text { expired volume in } 1 \text { second; } \mathrm{PEFR} \text {, peak expiratory flow rate. } \\
{ }^{a} \mathrm{P}<0.05 \text { between conditions }\end{array}$} \\
\hline
\end{tabular}


Heavy load carriage in females.

Table 3. Mean $( \pm$ SD) Size-matched comparison of male and female graded exercise test results. (Male data from Phillips et al. 2016b)

\begin{tabular}{|c|c|c|}
\hline Variable & Male $(n=7)$ & Female $(n=7)$ \\
\hline Age (y) & $25(3)$ & $24(5)$ \\
\hline Height (cm) & $176.9(3.6)$ & $176.7(4.5)$ \\
\hline Mass (kg) & $69.9(4.8)$ & $69.7(5.8)$ \\
\hline $\operatorname{BSA}\left(\mathrm{m}^{2}\right)$ & $1.9(0.1)$ & $1.8(0.1)$ \\
\hline$\dot{\mathrm{V}} \mathrm{O}_{2 \text { peak }}\left(\mathrm{L} \cdot \mathrm{min}^{-1}\right)-$ Unloaded & $3.66(0.37)$ & $2.94(0.39)^{a}$ \\
\hline$\dot{\mathrm{V}} \mathrm{O}_{2 \text { peak }}\left(\mathrm{L} \mathrm{min}^{-1}\right)-$ Loaded & $3.50(0.32)$ & $2.88(0.48)^{a}$ \\
\hline$\Delta \dot{\mathrm{V}} \mathrm{O}_{2}\left(\mathrm{Lmin}^{-1}\right)$ & $0.16(0.13)$ & $0.06(0.13)$ \\
\hline$\dot{\mathrm{V}}_{\text {Epeak }}\left(\mathrm{L}_{\mathrm{min}}{ }^{-1}\right)-$ Unloaded & $148.2(12.3)$ & $117.4(12.6)^{a}$ \\
\hline$\dot{\mathrm{V}}_{\text {Epeak }}\left({\left.\mathrm{L} \cdot \mathrm{min}^{-1}\right)- \text { Loaded }}^{-1}\right.$ & $144.2(7.8)$ & $115.1(17.8)^{a}$ \\
\hline$\Delta \dot{\mathrm{V}}_{\text {Epeak }}\left(\mathrm{L} \cdot \mathrm{min}^{-1}\right)$ & $4.0(11.6)$ & $2.3(12.2)$ \\
\hline Test Duration (s) - Unloaded & $1358(155)$ & $1186(118)^{a}$ \\
\hline Test Duration (s) - Loaded & $923(111)$ & $780(110)^{a}$ \\
\hline$\Delta$ Test Duration (s) & $435(89)$ & $406(47)$ \\
\hline$\Delta$ Test Duration $(\%)$ & $32(5)$ & $34(4)$ \\
\hline
\end{tabular}

BSA, body surface area; $\dot{\mathrm{V}}{ }_{2}$, oxygen consumption; $\dot{\mathrm{V}}_{\mathrm{E}}$, minute ventilation.

${ }^{a} \mathrm{p}<0.05$ between male and female groups. 
Heavy load carriage in females.

Table 4. Mean $( \pm \mathrm{SE})$ selected physiological and perceptual responses during prolonged exercise (Part II) in unloaded and loaded conditions. $\mathrm{n}=14$

\begin{tabular}{|c|c|c|c|c|c|c|}
\hline & \multicolumn{3}{|c|}{ Unloaded } & \multicolumn{3}{|c|}{ Loaded } \\
\hline & $5 \mathrm{~min}$ & $25 \mathrm{~min}$ & $45 \mathrm{~min}$ & $5 \min$ & $25 \mathrm{~min}$ & $45 \mathrm{~min}$ \\
\hline $\begin{array}{l}\text { Physiological } \\
\dot{\mathrm{VO}_{2}}\left(\mathrm{~L} \cdot \mathrm{min}^{-1}\right)\end{array}$ & $1.86(0.06)$ & $1.96(0.06)^{b}$ & $1.97(0.06)$ & $1.88(0.06)$ & $1.99(0.06)^{b}$ & $2.03(0.06)$ \\
\hline$\dot{\mathrm{V} C O}{ }_{2}\left(\mathrm{~L} \cdot \mathrm{min}^{-1}\right)$ & $1.72(0.06)$ & $1.80(0.07)^{b}$ & $1.81(0.06)$ & $1.74(0.04)$ & $1.86(0.04)^{b}$ & $1.87(0.04)$ \\
\hline RER & $0.92(0.01)$ & $0.92(0.01)$ & $0.92(0.01)$ & $0.93(0.01)$ & $0.94(0.01)$ & $0.92(0.01)$ \\
\hline HR (beats $\cdot \min ^{-1}$ ) & $152(3)$ & $166(4)^{b}$ & $172(4)^{c}$ & $156(3)^{a}$ & $170(3)^{a, b}$ & $177(2)^{a, c}$ \\
\hline $\mathrm{P}_{\mathrm{ET}} \mathrm{CO}_{2}(\mathrm{mmHg})$ & $38.2(0.9)$ & $35.3(1.0)^{b}$ & $33.5(1.0)^{c}$ & $36.5(1.0)$ & $33.6(1.1)^{a, b}$ & $32.9(1.2)^{a, c}$ \\
\hline Systolic BP (mmHg) & $141(3)$ & $143(4)$ & $144(4)$ & $138(3)$ & $141(2)$ & $143(3)$ \\
\hline Diastolic BP (mmHg) & $74(2)$ & $74(2)$ & $74(2)$ & $74(2)$ & $74(2)$ & $75(2)$ \\
\hline Core temperature $\left({ }^{\circ} \mathrm{C}\right)$ & $37.38(0.08)$ & $38.18(0.06)^{b}$ & $38.49(0.09)^{c}$ & $37.38(0.07)$ & $38.15(0.11)^{b}$ & $38.45(0.11)^{c}$ \\
\hline $\begin{array}{l}\text { Perceptual } \\
\text { Exercise stress }\end{array}$ & $2.4(0.2)$ & $3.7(0.4)^{b}$ & $4.9(0.6)^{c}$ & $2.9(0.2)^{a}$ & $5.0(0.5)^{a, b}$ & $6.0(0.5)^{a, c}$ \\
\hline Breathing Discomfort & $1.8(0.3)$ & $2.6(0.5)^{b}$ & $3.1(0.7)^{c}$ & $2.0(0.4)$ & $3.6(0.5)^{a, b}$ & $4.5(0.7)^{a, c}$ \\
\hline Leg fatigue & $2.8(0.4)$ & $4.1(0.5)^{b}$ & $4.6(0.6)^{c}$ & $2.9(0.2)$ & $5.4(0.5)^{a, b}$ & $6.2(0.5)^{a, c}$ \\
\hline $\begin{array}{l}\mathrm{VO}_{2} \text {, oxygen consumpt } \\
\text { end-tidal carbon dioxid } \\
{ }^{a} \mathrm{P}<0.05 \text { between condi } \\
{ }^{b} \mathrm{P}<0.05 \text { different betw } \\
{ }^{c} \mathrm{P}<0.05 \text { different betwe }\end{array}$ & 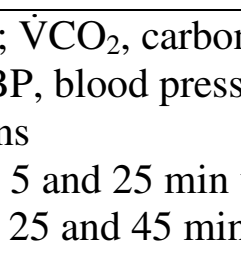 & $\begin{array}{l}\text { oxide producti } \\
\text { in condition } \\
\text { thin condition }\end{array}$ & RER, respira & exchange rat & , heart rate; $\mathrm{P}_{\mathrm{E}}$ & $\mathrm{O}_{2}$, pressure of \\
\hline
\end{tabular}


Heavy load carriage in females.

Table 5. Mean $( \pm \mathrm{SE})$ maximal inspiratory and expiratory respiratory muscle pressure measurements before (pre) and after (post) unloaded and loaded exercise $(n=14)$.

\begin{tabular}{lcc}
\hline Variable & Unloaded & Loaded \\
\hline MIP pre $\left(\mathrm{cm} \mathrm{H}_{2} \mathrm{O}\right)$ & $112(5)$ & $114(5)$ \\
MIP post $\left(\mathrm{cm} \mathrm{H}_{2} \mathrm{O}\right)$ & $111(6)$ & $103(6)^{a, b}$ \\
MEP pre $\left(\mathrm{cm} \mathrm{H}_{2} \mathrm{O}\right)$ & $136(8)$ & $135(8)$ \\
MEP post $\left(\mathrm{cm} \mathrm{H}_{2} \mathrm{O}\right)$ & $135(8)$ & $135(8)$ \\
\hline
\end{tabular}

MIP, maximal inspiratory pressure; MEP, maximal expiratory pressure.

${ }^{a} \mathrm{P}<0.05$ between unloaded-MIP and loaded-MIP post-exercise

${ }^{b} \mathrm{P}<0.05$ between MIP pre- and post-exercise within the loaded condition 
Heavy load carriage in females.

\section{Figure Captions}

Figure 1. Mean $( \pm \mathrm{SE}) \dot{\mathrm{V}} \mathrm{O}_{2}$, oxygen demand $(\mathrm{A}) ; \dot{\mathrm{V}}_{\mathrm{E}}$, minute ventilation $(\mathrm{B}) ; \dot{\mathrm{V}}_{\mathrm{A}}$, alveolar ventilation $(C) ; \dot{V}_{D}$, deadspace ventilation (D); $f_{B}$, breathing frequency $(E) ; V_{T}$, tidal volume (F) during exercise in loaded (closed circles) and unloaded (open circles) conditions. Lower case letter " $a$ " indicates significant difference $(\mathrm{P}<0.05)$ between conditions; lower case letter " $b$ " indicates significant difference between 5 and 45 min within conditions. $n=14$

Figure 2. Mean $( \pm \mathrm{SE})$ operating lung volume in loaded (closed symbols) and unloaded (open symbols) conditions, shown as a percentage of the measured forced vital capacity. Lower case letter " $a$ " indicates a significant difference $(\mathrm{P}<0.05)$ between conditions. $\mathrm{n}=14$ 

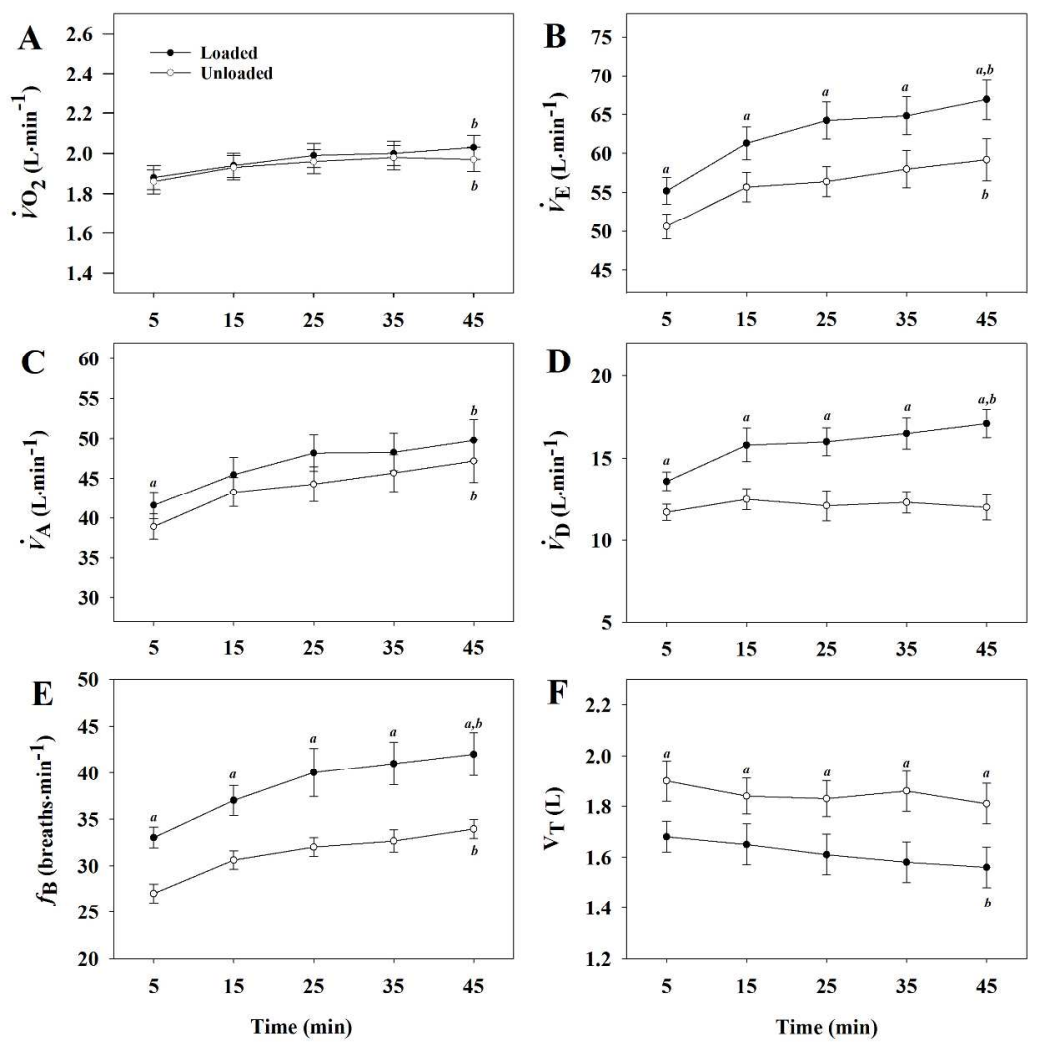

$257 \times 217 \mathrm{~mm}(300 \times 300 \mathrm{DPI})$ 


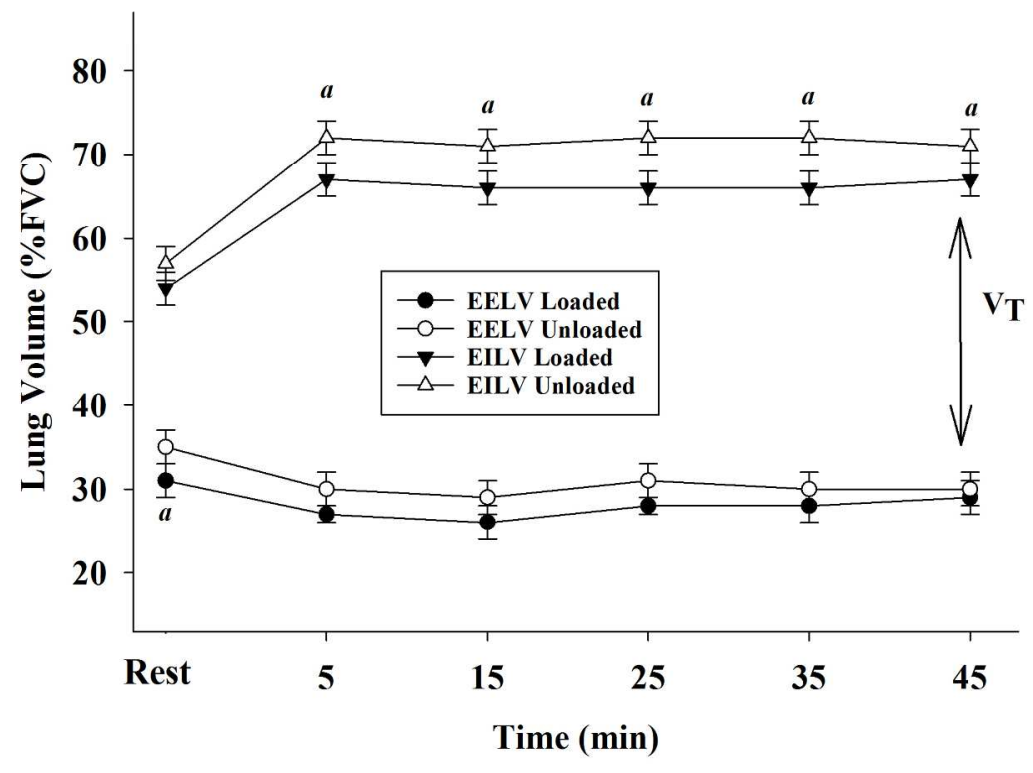

$203 \times 131 \mathrm{~mm}(300 \times 300 \mathrm{DPI})$ 